\title{
Migración y derechos humanos: la crisis de los Rohingya
}

\author{
Verónica Flores ${ }^{1}$ \\ UBA - CONICET - C.A.R.I.
}

El proceso de globalización ${ }^{2}$ se ha traducido entre otros en una mayor movilidad de población (lo cual motivó que el fenómeno migratorio sea tema de la agenda internacional desde hace ya varios años) y al mismo tiempo, entre otras cosas y por eso mismo, sitúe en el centro de la escena al tema de la diversidad cultural.

En ese contexto, de acuerdo con lo señalado por las Naciones Unidas, en 2015, hubo 244 millones de migrantes internacionales en el mundo, que representan el 3,3 \% de la población mundial, siendo Europa y Asia residencia de dos tercios de esos migrantes internacionales, y Asia-hacia-Asia, el mayor corredor regional en términos migratorios (United Nations, 2016).

El fenómeno migratorio ${ }^{3}$, como sabemos, abre un complejo dispositivo de regulación por parte de los estados, a su interior y fuera de sus límites, estrechamente relacionado a la construcción social de aquellos considerados "Otros" y evidencia, en esas regulaciones cuestiones relativas a las identidades y el elemento de poder propio de las relaciones sociales (Restrepo, 2010).

En el caso particular que nos ocupa, que ha cobrado una mayor visibilidad en los últimos tiempos, se encuentran los Rohingya, islámicos originarios del estado de Rakhine, en Myanmar, aunque la posición oficial de ese país es la de que los Rohingyas son inmigrantes ilegales llegados a Rakhine (o Arakan), tras la independencia de Burma, en 1948 o después de la guerra de liberación de Bangladesh, en 1971. Este grupo parece haberse asentado en ese estado desde el siglo XV y Buchanan sugirió en 1799 que los dos grupos nativos de Arakan eran los Rooinga y los Yakein (Ibrahim, 2016).

Hacia la Segunda Guerra Mundial, las masacres sucedidas en Arakan, en 1942, tuvieron que ver con la violencia comunal entre el ejército británico, los Rohingya y los habitantes de ese estado que eran budistas, deviniendo la región en una zona de polarización étnica.

\footnotetext{
${ }^{1}$ Licenciada en Historia (FFyL, UBA) y Doctoranda en Historia y Teoría de las Artes (FFyL, UBA). Es becaria CONICET e investigadora y Profesora Adjunta regular (USAL). Es miembro del Grupo de Trabajo sobre India y Asia del Sur, del Comité de Asuntos Asiáticos del Consejo Argentino para las Relaciones Internacionales (C.A.R.I.) y autora de distintas publicaciones.

2 Beck (1998) la entiende como un proceso, que crea vínculos y espacios sociales transnacionales, revaloriza culturas locales y trae a primer plano terceras culturas. Agrega que deben considerarse en él tres parámetros: a) un mayor espacio, b) la estabilidad en el tiempo y c) la densidad social de los entramados, interconexiones y corrientes icónicas transnacionales.

${ }^{3}$ Pueden consultarse consideraciones generales sobre el fenómeno en Rocha Trindade (1995).
} 
Tras la independencia de Burma, en 1948, la rebelión mujahideen se inició como un movimiento separatista que buscaba terminar con la discriminación a manos de la administración budista y se extendió hacia la década del '60, junto con el movimiento independentista del estado, a través de los budistas de la zona. En ese clima de hostilidad mutua, en 1982, el gobierno del General Ne Win estableció la Ley de Ciudadanía de Burma, denegándosela a los Rohingya, dejando a este grupo como una población sin estado (Constantine, 2012).

Mientras, en 2013, puede decirse que la población de los Rohingya ascendía a 1.23 millones de personas aproximadamente. A pesar de que la mayoría reside en el estado Rakhine de Myanmar, otros se han desplazado hacia Bangladesh, Pakistán y Tailandia, huyendo de la intolerancia de los ultra nacionalistas budistas y el acoso de las fuerzas de seguridad. Al mismo tiempo, su caso ha recibido atención internacional en relación con distintas circunstancias que se han ido sucediendo, a saber: los disturbios en Estado de Rakhine, en 2012 -que hicieron que el Presidente, Thein Sein, declarara el estado de emergencia y movilizara a las fuerzas de seguridad para restaurar el orden, evitando extender el clima hostil al resto de la nación que había desarrollado recientes reformas democráticas, terminando con un largo período de gobierno militar- (Reuters, 2012; The Guardian, 2012) y otros incidentes violentos en 2013 y 2014 (BBC News, 2014), la crisis de refugiados Rohingya en 2015 (el gobierno tailandés comenzó a perseguir a los contrabandistas que tradicionalmente llevaban a los Rohingya a campamentos en el sur de Tailandia y, a resultas de ello, los contrabandistas los abandonaban a su suerte en el mar, sin que los países vecinos se dispusieran a ayudar) y la represión militar en 2016 (Perlez \& Moe, 2016).

En 2012, el entonces Embajador de los Estados Unidos en Myanmar señaló lo que considera el racismo en la sociedad del país; sin embargo otros, como Maung Zarni (2012) han planteado la cuestión de la violencia comunal como una directa consecuencia de las tácticas del anterior régimen militar (en cuanto a dividir y reinar), no modificadas sustancialmente por el régimen de Thein Sein. A lo largo de la historia, a más del conflicto entre los budistas ultra nacionalistas y los Rohingya (Ilamados muchas veces "islámicos bengalíes", en un tono peyorativo que busca desconocer su nacimiento en el país y remitir su origen a Bangladesh) (The Indian Express, 2017), puede agregarse que hacia 1967, Ne Win culpó a los "ambiciosos comerciantes chinos" por el fracaso de sus medidas económicas, lo que derivó en distintos episodios en contra de los chinos en el país. A ello se suma la mala percepción que los militares tienen de los habitantes del estado de Rakhine y el movimiento etnonacional del que participan algunos, buscando la autonomía política de ese estado.

Durante el gobierno militar del país, los movimientos y la vida de los Rohingya fueron restringidos y ese abuso se extendió al logro de afluencia de dinero de esa población a las fuerzas de seguridad, por distintos motivos. Tal hecho fue resentido por el resto de la población de Rakhine, que parecía percibir un cierto favoritismo de la gestión hacia los Rohingya. Por otro lado, el trato desigual a los Rohingya se manifiesta también en el número desproporcionado de los mismos en el trabajo forzado, mayor que el de cualquier otro grupo étnico en el país. 
Esta situación llevó a estallidos de violencia en contra de los Rohingya, por parte de los civiles, a los cuales se sumó la acción de las fuerzas de seguridad. Todo lo mencionado sería relatado en el informe de Human Rights Watch (2013), describiendo el desplazamiento forzado de más de 125.000 Rohingya y otros islámicos, en una terrible crisis humanitaria. De acuerdo al informe, los dos grupos más activos en la organización de sucesivos ataques a islámicos Rohingya y no Rohingya fueron la orden local de monjes budistas y el poderoso Partido del Desarrollo de las Nacionalidades de Rakhine, fundado en el año 2010 por nacionalistas de ese estado (Human Rights Watch, 2013).

Mientras los países de la región no quieren atender a los problemas y crisis humanitaria de los Rohingya, puede decirse que Tailandia sostiene que ha ayudado a las personas que encontraron en botes y permite campos en sus costas, pero, sin embargo, se sabe que busca ahuyentar a nuevos botes con refugiados y no desea asentamientos permanentes. Malasia, por su parte, ha dado la orden a su armada de repeler nuevas llegadas de esta población, aunque ese país es el destino más buscado por esta población, debido a su mayoría islámica. En Bangladesh, por otro lado, se estima que hay alrededor de 200.000 viviendo en sus campos de refugiados y mientras por momentos ese país permite el acceso a los mismos, en otros momentos, los envía de regreso a Myanmar. Indonesia no desea recibir Rohingya y les ha pedido a sus pescadores que no rescaten más personas en el mar. Finalmente, algunos de los Rohingya han llegado a Jammu y Cachemira. Hay ya 14.000 refugiados registrados en esa zona, en India y su asentamiento en esa zona sensible despierta resquemores internos y de alcance internacional ${ }^{4}$ (BBC News, 2015; The Indian Express, 2017).

La situación, que ha sido caracterizada por un oficial de Naciones Unidas como de "limpieza étnica de los Rohingya" (Hindustan Times, 2016), adquiere entonces una dimensión regional más amplia, considerando el número de Rohingya que huyó y huye a los países mencionados, y las terribles circunstancias asociadas a ello, como, por ejemplo, la trata de personas (European Comission, 2016), junto a la posible amenaza de contactos con grupos radicales.

Finalmente, aun cuando es cierto que Myanmar $^{5}$ se encuentra bajo presión internacional por el tema y que su gobierno ha solicitado "tiempo y espacio" para resolver esta crisis (Hindustan Times, 2017), se requiere de una urgente atención de la comunidad internacional que permita ayudar a priorizar el diálogo inter-comunal y la mediación para la resolución del conflicto en el Estado de Rakhine, mientras se aborda la provisión de necesidades básicas a la población afectada, buscando alcanzar soluciones no solamente urgentes sino fundamentalmente duraderas, que permitan asegurar el respeto a los derechos humanos de todos los implicados, de manera particular de la población Rohingya y no sumar un conflicto ampliado a la región.

\footnotetext{
${ }^{4}$ India advirtió recientemente a Myanmar que militantes de Lashkar-e-Taiba, asentados en Pakistán buscaban explotar la radicalización en la comunidad Rohingya, lo cual amenaza la seguridad no solamente de India y Myanmar sino de toda la región. De hecho, el fundador del grupo Lashkar-e-Taiba, Hafiz Saeed, acusó a India de ser cómplice de Myanmar en su persecución de los Rohingya (Jha, 2017).

${ }^{5}$ Su actual (y primer) Consejera de Estado es Aung San Suu Kyi, quien recibiera el Premio Nobel de la Paz en 1991, al igual que otros premios y honores, atendiendo a su lucha no violenta por la democracia y los derechos humanos (Nobelprize.org, s.f.).
} 


\section{Referencias Bibliográficas:}

BBC NEWS (2015). "Why are so many Rohingya migrants stranded at sea?" Asia (18 de mayo). Recuperado de http://www.bbc.com/news/world-asia-32740637

BBC NEWS (2014). "Why is there communal violence in Myanmar?". Asia (3 de julio). Recuperado de http://www.bbc.com/news/world-asia-18395788

BECK, U. (1998). ¿Qué es la globalización? Falacias del globalismo, respuestas a la globalización. Barcelona: Edit. Paidós.

CONSTANTINE, G. (2012). Between Burma and Bangladesh: Rohingya, a Stateless People. Pulitzer Center (18 de abril). Recuperado de http://pulitzercenter.org/reporting/between-burma-and-bangladesh-rohingya-stateless-people

EUROPEAN COMMISSION (2016). Humanitarian Aid and Civil Protection. The Rohingya crisis. Recuperado de http://ec.europa.eu/echo/files/aid/countries/factsheets/rohingya en.pdf

HINDUSTAN TIMES (2017). “Myanmar asks for 'time and space' to solve Rohingya Muslim crisis" (23 de enero). Recuperado de http://www.hindustantimes.com/world-news/myanmar-asks-for-time-and-space-to-solve-rohingyamuslim-crisis/story-uGWcaUtVOqFdq0VW4QPGoN.html

HINDUSTAN TIMES (2016). “Myanmar pursuing 'ethnic cleansing' of Rohingya: UN official” (25 de noviembre). Recuperado de http://www.hindustantimes.com/india-news/persecuted-in-myanmar-rohingya-muslims-find-newhome-in-jammu/story-NOv2VcEw2PyNW4Y28E1w7M.html

HUMAN RIGHTS WATCH (2013). "'All You Can Do is Pray': Crimes Against Humanity and Ethnic Cleansing of Rohingya Muslims in Burma's Arakan State". Recuperado de https://www.hrw.org/report/2013/04/22/all-you-can-dopray/crimes-against-humanity-and-ethnic-cleansing-rohingya-muslims

IBRAHIM, A. (2016). The Rohingyas: Inside Myanmar's Hidden Genocide. New York: Oxford University Press.

JHA, P. (2017). "Lashkar militants inciting Rohingya refugees, India warns Myanmar". Hindustan Times (7 de febrero). Recuperado de http://www.hindustantimes.com/india-news/india-tells-myanmar-rohingya-issue-ticking-bomb-willcome-back-to-haunt-region/story-m7b2QFQn13uzOfX4nK9PWI.html 
NOBELPRIZE.ORG (s.f.). Aung San Suu Kyi - Biographical. Recuperado de http://www.nobelprize.org/nobel_prizes/peace/laureates/1991/kyi-bio.html

PERLEZ, J. \& MOE, W. (2016). "Violence Escalates Between Myanmar Forces andRohingya”. The New York Times. Asia Pacific (13 de noviembre). Recuperado de https://www.nytimes.com/2016/11/14/world/asia/violence-escalatesbetween-myanmar-forces-and-rohingya.html?_r=0

RESTREPO, E. (2010). Identidad: apuntes teóricos y metodológicos. En Castellanos Llanos, G; Grueso, D. I. \& Rodriguez, M. -coord.- Identidad, cultura y política. Perspectivas conceptuales, miradas empíricas (pp. 61-70). México: Honorable Cámara de Diputados-Universidad del Valle-Miguel Ángel Porrúa.

REUTERS (2012). "Four killed as Rohingya Muslims riot in Myanmar: government". World News (9 de junio). Recuperado de http://www.reuters.com/article/us-myanmar-violence-idUSBRE85714E20120608

ROCHA-TRINDADE, M. B. (1995). Sociologia das Migraçoes. Lisboa: Universidade Aberta.

THE GUARDIAN (2012). "Burma ethnic violence escalates as villagers flee". South and Central Asia (12 de junio). Recuperado de https://www.theguardian.com/world/2012/jun/12/burma-ethnic-violence-escalates

THE INDIAN EXPRESS (2017). "The Most Unwanted: A gripping account of Rohingya refugees living in India" (8 de enero). Recuperado de http://indianexpress.com/article/india/the-most-unwanted-a-gripping-account-of-rohingyarefugees-living-in-india-4464103/

UNITED NATIONS, Department of Economic and Social Affairs, Population Division (2016). International Migration Report 2015. New York: United Nations. Retrieved from

http://www.un.org/en/development/desa/population/migration/publications/migrationreport/docs/MigrationReport 2015.pdf\#page $=$

ZAMI, M. (2012). "The real culprits behind the violence in Rakhine state". The Nation (29 de Agosto). Recuperado de http://www.nationmultimedia.com/news/opinion/aec/30189267 